\title{
Monte Carlo Simulations for the Study of a Moderated Neutron Detector
}

\author{
M. B. Gomez Hornillos, ${ }^{*}$ V. Gorlychev, R. Caballero, G. Cortes, A. Poch, C. Pretel and F. Calvino \\ Seccio d'Enginyeria Nuclear, Universitat Politecnica de Catalunya, 08028, Barcelona, Spain \\ J. L. Tain, A. Algora and J. Agramunt \\ IFIC, CSIC-Univ. Valencia, Valencia, Spain \\ D. Cano-Ott, T. Martinez and E. Mendoza \\ CIEMAT-Madrid, Spain
}

J. Rissanen, J. Aysto, A. Jokinen, T. Eronen, I. Moore and H. Penttila

JYFL, Jyväskylä, Finland

(Received 26 April 2010)

\begin{abstract}
This work presents the Monte Carlo simulations performed with the MCNPX and GEANT4 codes for the design of a BEta deLayEd Neutron detector, BELEN-20. This detector will be used for the study of beta delayed neutron emission and consists of a block of polyethylene with dimensions $90 \times 90 \times 80 \mathrm{~cm}^{3}$ and 20 cylindrical ${ }^{3} \mathrm{He}$ gas counters. The results of these simulations have been validated experimentally with a ${ }^{252} \mathrm{Cf}$ source in the laboratory at UPC, Barcelona. Also the first experiment with this detector has been carried out in November 2009 in JYFL, Finland. In this experiment the neutron emission probability after beta decay of the fission products ${ }^{88} \mathrm{Br}$, ${ }^{94,95} \mathrm{Rb}$, and ${ }^{138} \mathrm{I}$ has been measured; this data is still under analysis. Simulations with MCNPX and GEANT4 have been performed in order to obtain the efficiency of the BELEN-20 detector for each of the above nuclei using the neutron energy distribution corresponding to each nucleus.
\end{abstract}

PACS numbers: 29.40.-n, 29.20.Hm

Keywords: Monte Carlo simulations, GEANT4, MCNPX, Beta delayed neutron emission, Neutron detector DOI: $10.3938 / \mathrm{jkps} .59 .1573$

\section{INTRODUCTION}

Monte Carlo simulations are a basic tool for the optimisation of the design of new detectors and experimental setups. Two of the most commonly used Monte Carlo simulation codes for the passage of particles through matter are GEANT4 [1] and MCNPX [2]. GEANT4 is a very versatile code and provides complete information on the particle track among other features. MCNPX is the simulation code that is most widely accepted by the neutron community. Both codes, GEANT4 (v.4.9.1.p2) and MCNPX (v.2.5.0), have been used in this work in a complementary way in order to design a detector to measure the probability of neutron emission after beta decay $\left(\mathrm{P}_{n}\right)$.

Neutron emission after beta decay plays a major role in the safe design of nuclear power plants [3]. Furthermore the $\mathrm{P}_{n}$ is a very valuable piece of data for nuclear astrophysics, in particular for the r-process, since it defines the decay path towards stability during freeze-out, and these emitted neutrons provide a source of late time

*E-mail: belen.gomez@upc.edu neutrons. Also, the $\mathrm{P}_{n}$ carries important nuclear structure information for nuclei very far from the valley of stability, where other measurements are not yet possible. However the existing experimental data nowadays is scarce and new high precision measurements are necessary to improve the data bases.

The aim of this work is to design an optimal detector to provide experimental data from experiments performed in existing and future new accelerator facilities.

\section{DETECTOR CHARACTERISTICS}

The main features of the BELEN-20 detector have been decided based on simulations with the MCNPX Monte Carlo code. The objective of these simulations was to obtain a device with a neutron detection efficiency as high and as constant as possible in the energy range of interest for $\beta$-delayed neutron emission (from a few $\mathrm{keV}$ to a few $\mathrm{MeV}$ ).

Based on the results of the MCNPX simulations the detector consists of a block of polyethylene surrounded by a shielding polyethylene layer with total dimensions $90 \times 90 \times 80 \mathrm{~cm}^{3}$ and a cylindrical beam hole in the 


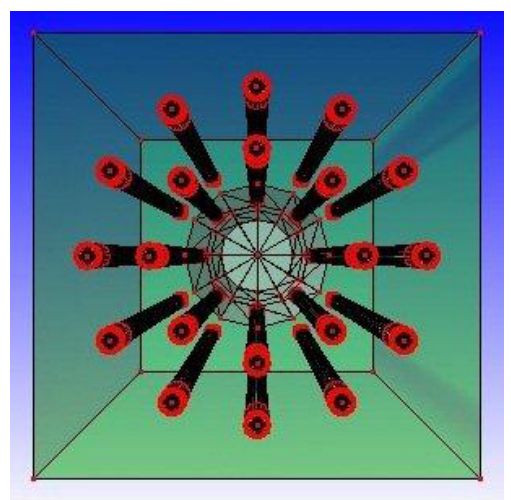

Fig. 1. (Color online) Scheme of the polyethylene matrix with the $20{ }^{3} \mathrm{He}$ counters in two crowns around the beam hole.

centre with a radius of $5 \mathrm{~cm}$ [4]. The central block has two concentric crowns of 8 and $12{ }^{3} \mathrm{He}$ gas cylindrical counters around the beam hole. The ${ }^{3} \mathrm{He}$ counters have a $60 \mathrm{~cm}$ effective length and $2.54 \mathrm{~cm}$ diameter and contain $20 \mathrm{~atm}$ of ${ }^{3} \mathrm{He}$ gas. The layout of the detector is presented in Fig. 1.

The detection of the neutrons is achieved via the detection of the charged particles that are produced in the reaction

$$
{ }^{3} \mathrm{He}+\mathrm{n} \longrightarrow{ }^{3} \mathrm{H}+\mathrm{p}+763.7 \mathrm{keV} .
$$

The cross section of this reaction increases with decreasing neutron energy [5] and therefore motivates the need for neutron moderation in the polyethylene in order to increase the neutron detection probability.

The request of having an efficiency curve as constant as possible for the neutron energy range of interest is due to the fact that the information on the initial energy of the emitted neutron will be lost because of the moderation in the polyethylene. Thus having a constant response curve reduces the uncertainty in the neutron emission probability.

\section{MONTE CARLO SIMULATIONS}

The efficiency curve obtained with MCNPX is presented in Fig. 2 along with the one obtained with GEANT4 in order to compare the results of both codes. It can be appreciated that the relative difference between MCNPX and GEANT4 is 7.5\% in average along the energy range from $1 \mathrm{keV}$ to $5 \mathrm{MeV}$, although the difference between both simulation codes reduces for high energy.

Regarding the simulation of neutron transport it is common knowledge among the neutron community that there are some discrepancies between the results obtained by GEANT4 and by the more widely accepted neutron transport simulation code MCNPX as it has been shown in Fig. 2. For this reason both codes have been used for the design of this neutron detector in a complementary way. MCNPX was used to decide the

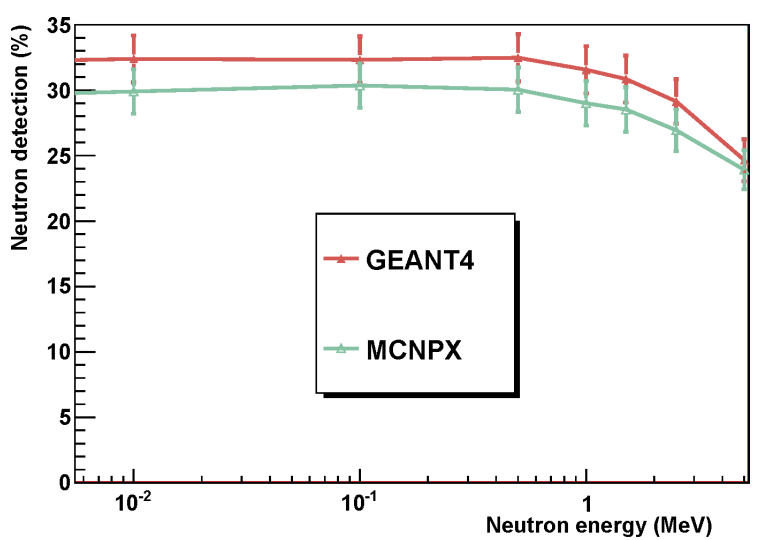

Fig. 2. (Color online) Neutron detection efficiency according to the Monte Carlo simulation codes GEANT4 and MCNPX.

best placement for the detectors in order to achieve constant neutron detection efficiency. GEANT4 was used to study the particles produced in the ensemble due to the passage of the neutron and the energy deposited by them in the ${ }^{3} \mathrm{He}$ gas. Keeping the discrepancy between GEANT4 and MCNPX in mind, the information provided by GEANT4 is of high interest for the design of the detector in order to look at aspects on which GEANT4 provides information more readily, such as the particles created in the ensemble due to the interactions, their energy deposition and full tracking information.

\section{GEANT4 simulations}

A GEANT4 simulation using the energy distribution of the neutrons from a ${ }^{252} \mathrm{Cf}$ source has been performed in order to obtain the shape of the spectrum of the deposited energy in the ${ }^{3} \mathrm{He}$ gas and study the energy deposition of particles other than protons and tritons in the gas. The distribution of the ${ }^{252} \mathrm{Cf}$ neutrons has been implemented as the Watt Spectrum from [7].

The spectrum of the energy deposited in the gas is presented in Fig. 3, it can be clearly appreciated that the influence of events other than the ones from neutron detection is not statistically significant and the noise can be discriminated since it occurs at low energies.

The time pattern of the appearance of the ${ }^{3} \mathrm{H}$ in the ${ }^{3} \mathrm{He}$ gas (i.e., detection of the neutron) is another main point of the simulations due to the long delay (hundreds of $\mu \mathrm{s}$ ) between the emission of the neutron and its detection in the ${ }^{3} \mathrm{He}$ caused by the neutron moderation process in the polyethylene matrix. The results of the simulation are shown in Fig. 4. This time has to be known in order to be able to correlate the decay of the precursor nucleus with the detected neutron in the experimental data. The data acquisition with this detector will be performed in a triggerless mode where all signals above an energy threshold will be stored with a time stamp [8]. The events will be reconstructed in the on/offline analysis, with full flexibility to modify the correlation time. The capability of being able to modify the correlation 


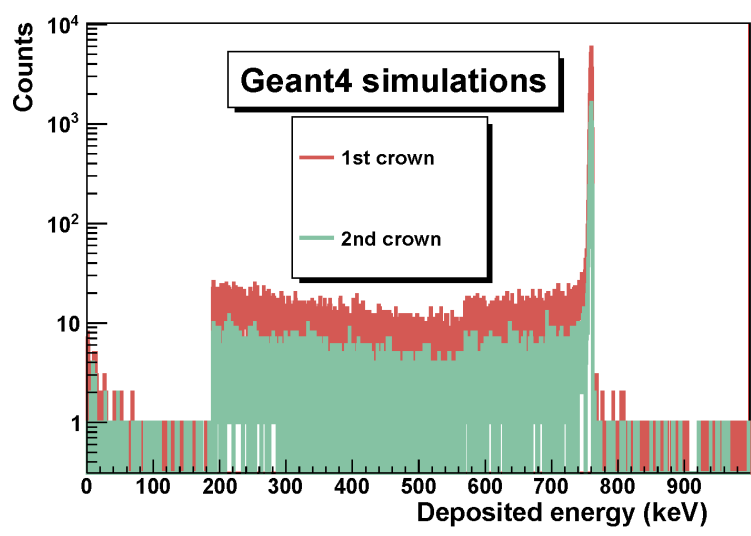

Fig. 3. (Color online) Energy deposited in the ${ }^{3} \mathrm{He}$ counters in each of the two crowns with a ${ }^{252} \mathrm{Cf}$ neutron source. The plot shows the wall effect counts above $191 \mathrm{keV}$ and the full energy deposition peak at $763.7 \mathrm{keV}$.



Fig. 4. (Color online) Time elapsed from the emission of a neutron to its detection in each of the two ${ }^{3} \mathrm{He}$ crowns with a ${ }^{252} \mathrm{Cf}$ neutron source.

time is key to an optimised background removal.

\section{SIMULATION VALIDATION}

\section{1. ${ }^{252}$ Cf test}

An experimental test has been carried out with a ${ }^{252} \mathrm{Cf}$ neutron source in the UPC laboratory in order to validate the different aspects of the Monte Carlo simulations, such as the efficiency, the energy deposited in the counter and the moderation time. At the time of the experiment, the ${ }^{252} \mathrm{Cf}$ source had an activity of $6.0 \pm$ $0.9 \mathrm{kBq}$ which corresponded to a neutron emission rate of $630 \pm 90 \mathrm{n} / \mathrm{s}$. The results of the efficiency of the neutron detector in this experimental test are presented in Table 1 along with the values of the GEANT4 and MCNPX simulation codes. The agreement between the experimental and the calculated efficiency is good, however it should be pointed out that the activity of the ${ }^{252} \mathrm{Cf}$ source has a $15 \%$ uncertainty.

Figure 5 shows the spectrum of the deposited energy in one counter from the inner crown and one from the outer crown. This figure can be compared to (Fig. 3), illustrating the good agreement of the shape predicted by
Table 1. Efficiency of the neutron detector with a ${ }^{252} \mathrm{Cf}$ source according to GEANT4, MCNPX and experiment.

\begin{tabular}{crrrr}
\hline \hline crown & GEANT4 $(\%)$ & MCNPX $(\%)$ & Experimental(\%) \\
\hline 1st crown & $25.0 \pm 1.6$ & $21.3 \pm 1.5$ & $21.3 \pm 3.2$ \\
2nd crown & $5.4 \pm 0.7$ & $6.0 \pm 0.8$ & $4.9 \pm 0.7$ \\
total & $30.4 \pm 1.7$ & $27.3 \pm 1.7$ & $26.1 \pm 3.9$ \\
\hline \hline
\end{tabular}

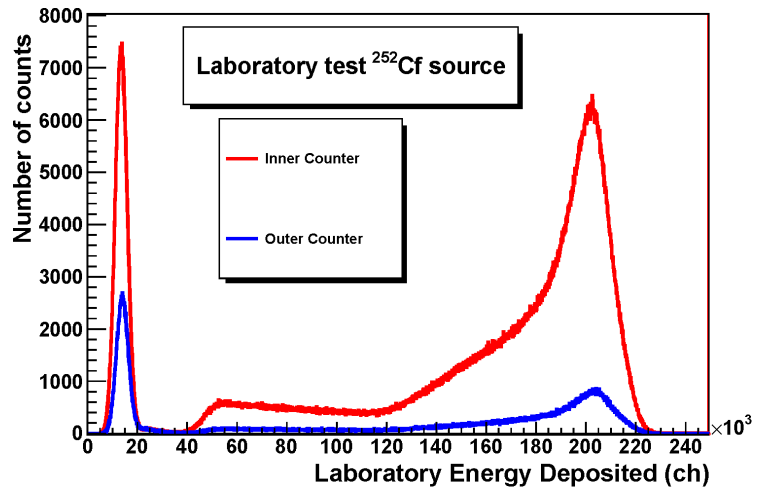

Fig. 5. (Color online) Energy deposited in a counter in the inner crown and a counter in the outer crown in the laboratory test at UPC with the ${ }^{252} \mathrm{Cf}$ source.

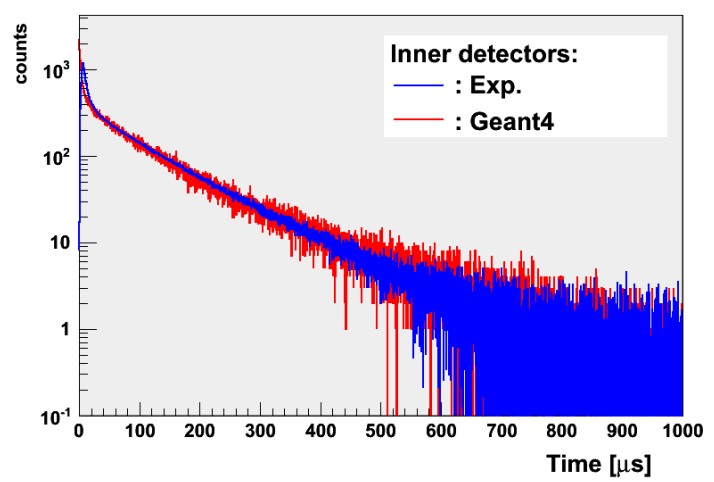

Fig. 6. (Color online) Time correlation between the neutron emission and the neutron detection (in the inner crown of detectors) for a ${ }^{252} \mathrm{Cf}$ source. GEANT4 simulations and experiment at UPC lab.

the GEANT4 simulations. Additionally it is clear from Fig. 5 that the noise can be easily discriminated since it occurs at energies below $191 \mathrm{keV}$. In the experimental spectrum (Fig. 5) there is a more marked $2^{\text {nd }}$ edge effect than in the one from the simulations (Fig. 3). This is due to the ballistic deficit since the time constant of the preamplifier was not large enough to collect the complete charge of the reaction in some cases.

Regarding the time correlation between the neutron emission and its detection, the comparison between the simulations and the experiment is presented in Fig. 6 for the inner crown and in Fig. 7 for the outer crown. The agreement was good in general, which confirmed that the triggerless data acquisition was working as expected, however there was some disagreement about the rising time between the simulations and the experiment. In 


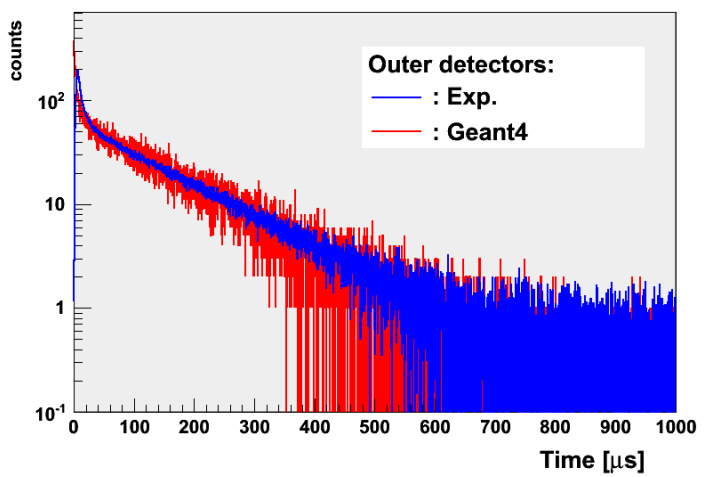

Fig. 7. (Color online) Same as Fig. 6 but for the outer crown of detectors.

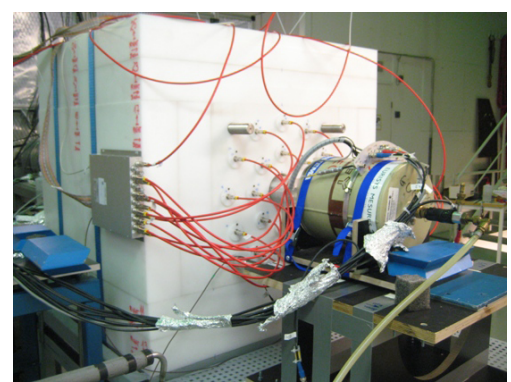

Fig. 8. (Color online) Setup in the experiment at the JYFLTRAP beam line with the neutron and Germanium detector.

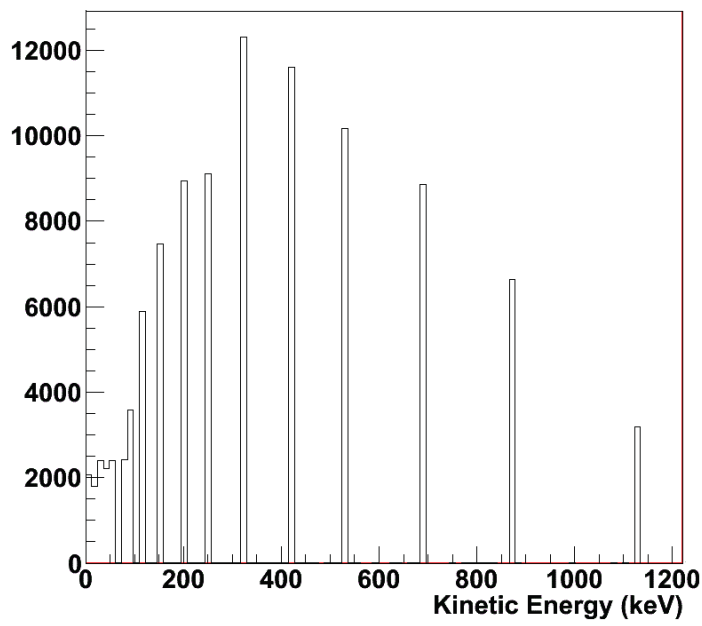

Fig. 9. Energy distribution of the beta delayed neutrons from ${ }^{94} \mathrm{Rb}$ according to ENDF7.

the beta delayed neutron emission experiments the correlation will be performed between the detection of the beta particle in a Si detector and the detection of the neutron in the ${ }^{3} \mathrm{He}$. In the laboratory test at UPC with the neutrons emitted by the ${ }^{252} \mathrm{Cf}$ source, the detection of the beta particle was substituted by the detection of the prompt gamma rays emitted in the fission of ${ }^{252} \mathrm{Cf}$.
Table 2. Efficiency of the neutron detector for the neutron energy distributions of the following nuclei.

\begin{tabular}{ccc}
\hline \hline Nucleus & GEANT4 $(\%)$ & MCNPX (\%) \\
\hline${ }^{88} \mathrm{Br}$ & $32.4 \pm 1.8$ & $30.2 \pm 1.7$ \\
${ }^{94} \mathrm{Rb}$ & $32.3 \pm 1.8$ & $30.0 \pm 1.7$ \\
${ }^{95} \mathrm{Rb}$ & $32.0 \pm 1.8$ & $30.1 \pm 1.7$ \\
${ }^{138} \mathrm{I}$ & $32.0 \pm 1.8$ & $30.1 \pm 1.7$ \\
\hline \hline
\end{tabular}

\section{Experiment at JYFL}

The first experiment using the BELEN-20 detector and its triggerless acquisition system was performed in November 2009 at JYFL, Finland. In this experiment the precursor nuclei were ${ }^{88} \mathrm{Br},{ }^{94,95} \mathrm{Rb}$, and ${ }^{138} \mathrm{I}$, all of them are fission products and neutron emitters after beta decay. Data is still under analysis. A picture of the setup is shown in Fig. 8. Simulations with MCNPX and GEANT4 have been used to predict the efficiency of the BELEN-20 detector for each of the nuclei above. The neutron energy distribution for each of the nuclei was obtained from ENDF7 [5]. These neutron energy distributions were implemented in the simulation codes as neutron sources. The shape of one of these distribution $\left({ }^{94} \mathrm{Rb}\right)$ is presented in Fig. 9 as an example since the energy distributions of the four nuclei studied here are quite similar and they all have their mean value below $500 \mathrm{keV}$. In the case of ${ }^{95} \mathrm{Rb}$ there was no ENDF7 [5] neutron energy distribution available and the values were obtained from [6]. The detection efficiencies of BELEN-20 obtained in the simulations with GEANT4 and MCNPX for each of the above fission products are presented in Table 2. Both simulation codes agree within the statistical error, being the average difference between the predictions of MCNPX and GEANT4 of 7\%. These values will be compared to the results obtained in the experiment at JYFLTRAP once the analysis is finished.

\section{REFERENCES}

[1] J. Allison et al., IEEE Trans. Nucl. Sci. 53, 1 (2006).

[2] MCNPX: https://mcnpx.lanl.gov.

[3] G. Rudstam et al., NEA/WPEC6 report, 2002.

[4] M. B. Gomez Hornillos, et al., Nucl. Instrum. Methods Phys. Res., Sect. A, in progress.

[5] M. B. Chadwick et al., Nucl. Data Sheets 107, 2931 (2006).

[6] R. C. Greenwood et al., Nucl. Sci. Eng. 91, 305 (1985).

[7] A. B. Smith et al., Phys. Rev. 108, 411 (1957).

[8] J. Agramunt et al., Nucl. Instrum. Methods Phys. Res., Sect. A, in progress. 